International Journal of Biomedicine | June 2021 - Volume 11, Issue Suppl_1: Abstracts from the Third Russian International Conference "Cryo-electron microscopy 2021: achievements and prospects"

POSTER ABSTRACT PRESENTATIONS

SESSION TITLE: STRUCTURE AND FUNCTIONS OF THE TRANSCRIPTION AND TRANSLATION APPARATUS OF THE CELL

DOI: 10.21103/JJBM.11.Suppl_1.P28

\title{
Abstract P-28: Structural Dynamics of DNA-Associated Chaperon Facilitates Chromatine Transcription
}

$\underline{\text { Olesya Volokh }^{1}}$, Anastasia Sivkina $^{1}$, Maria Karlova ${ }^{1}$, Elena Kotova ${ }^{2}$, Vasily Studitsky ${ }^{1,2}$, Olga Sokolova ${ }^{1}$

${ }^{1}$ Biology Faculty, Lomonosov Moscow State University, Moscow, Russia ${ }^{2}$ Fox Chase Cancer Center, Philadelphia, Pennsylvania, USA

Background: Histone chaperon FACT ("FAcilitates Chromatin Transcription") is a multifunctional and conserved eukaryotic protein involved in DNA transcription, replication and repair; which can reversibly unfold nucleosomes in presence of ATP. FACT is necessary for the viability and growth of breast tumor cells meanwhile in normal cells it can be knocked out without loss of vitality. Human FACT (hFACT) is a target for promising anticancer drug curaxins, which causes FACT trapping in chromatin of cancer cells and destabilizes the nucleosome. The nucleosome-unfolding activity is an important function of hFACT in vivo; however, the mechanism of FACT-dependent nucleosome unfolding remains unknown.

Methods: Here, we studied negative stained hFACT structure using single particle electron microscopy using JEOL 2100 TEM. Micrographs were captured with 25k magnification, and $4.1 \AA$ pixel size. EM images preprocessing and single particles collection were performed in EMAN2.3, followed by 2D-particles analysis in RELION2.0. Final 2D-classes included $\sim 70$ 000 single particles images.

Results: Based on 2D-classess data analysis we evaluated several states of hFACT reflecting its conformational flexibility: the "closed" complex is characterized by four domains localized close to each other and forming a compact structure; "intermediate" state represented by classes with identified three domains having compact structure and more disordered fourth domain, and the "open" complex, represented by three domains forming almost linear structure. The "closed" and "open" states are present in comparable amounts and 
significantly outnumber the "intermediate" state. It has been shown that hFACT domains are connected through flexible linkers and SPT16 and SSRP1 dimerization domains (DDs) form the "joint"-like connection between the two subunits. In the "closed" conformation, the DNA-binding surface of FACT is covered by its two C-terminal and middle domains (MDs). The N-terminal domain (NTD) of SPT16 was not resolved previously, but it is the best candidate for the forth domain that is clearly visible only in the "closed" conformation of hFACT, based on its dimensions and the longest linker length.

Conclusion: We propose that during conversion to the "open" complexes SPT16 NTD is moving away from the other subunits leading to formation of the first intermediate state with the NTD domain poorly resolved or not resolved, while less mobile DDs and MDs maintain more compact structure and the DNAbinding site is still protected by the CTDs. In the "open" state SPT16/SSRP1 visible MDs and DDs form almost linear structure, unmasking the DNA-binding sites and making them accessible for the interaction with a nucleosome.

Key Words: transcription $・$ nucleosome $・$ FACT

This work was supported by the Russian Science Foundation (Grant No. 1974-30003). Electron microscopy was performed on Unique scientific installation '3D-EMC.'

*Corresponding author: Olesya Volokh.E-mail: olesyavolokh@gmail.com

International Journal of Biomedicine. 2021;11 Suppl 1: S24.

doi: 10.21103/IJBM.11.Suppl_1.P28

(C)2021 International Medical Research and Development Corporation 